\title{
Las mujeres que se quedan: migración e implicación en los procesos de búsqueda de atención de servicios de salud
}

\author{
Marta Caballero, Soc, Comunic, D en C Soc, (') René Leyva-Flores, MD, Dr en Soc,(1) \\ Sandra Catalina Ochoa-Marín, Enf, Dra en SP,(2) Ángel Zarco, Antr, ${ }^{(3)}$ Claudia Guerrero, Etnól, M en Antrop de la Med y Sal Int. ${ }^{(4)}$
}

\author{
Caballero M, Leyva-Flores $\mathbf{R}$, \\ Ochoa-Marín SC, Zarco A, Guerrero C. \\ Las mujeres que se quedan: \\ migración e implicación en los procesos \\ de búsqueda de atención de servicios de salud. \\ Salud Publica Mex 2008;50:24I-250.
}

\section{Resumen}

Objetivo. Analizar los procesos de búsqueda de atención de los servicios de salud en comunidades de alto índice migratorio. Material y métodos. Estudio cualitativo, realizado entre octubre de 2004 y mayo de 2005 en dos comunidades de alto índice de intensidad migratoria internacional en México. Se entrevistó a 60 mujeres compañeras de migrantes en dos comunidades (urbana/rural) del centro de México con elevada migración hacia Estados Unidos. Resultados. En el contexto de la migración se identifican dos formas de recomposición familiar: integración de la mujer a su familia y casos de mujeres solas. La primera mostró mayor apoyo y control para buscar atención médica, sobre todo en salud sexual y reproductiva. La segunda tiene menor apoyo, pero mayor capacidad para buscar atención médica, con privacidad e independencia. Conclusiones. La reestructuración familiar condiciona la búsqueda oportuna de servicios de salud en mujeres compañeras de migrantes. Las situaciones identificadas funcionan como obstáculos o facilitadores de la movilización de recursos para la atención de la salud.

Palabras clave: migración; familia; salud; mujer; México
Caballero M, Leyva-Flores R,

Ochoa-Marín SC, Zarco A, Guerrero C.

Women who are left behind:

the impact of international migration

on the process of seeking health care.

Salud Publica Mex 2008;50:24I-250.

\begin{abstract}
Objective.To analyze how women who are left behind seek care in communities with a high migration index. Material and Methods. This is a cross-sectional study which used a qualitative approach. Sixty in-depth interviews were conducted between October 2004 and May 2005 with female partners of migrants in two communities (urban / rural) from a region in central Mexico with high migration to the United States. Results. Migration determines two forms of familial reorganization: one in which female partners of migrants reintegrate with their families; and one in which they remain on their own. Women who reintegrate with their families receive more support, but are also subjected to more control, rendering them with less capability to seek care, particularly related to sexual and reproductive health. Women who remain on their own have less support but have more control and capacity to seek care, with privacy and independence. Conclusions. The familial reorganization influences timely health care-seeking behaviour of female partners of migrants. The situations identified can function as obstacles or facilitators to mobilize healthcare resources.
\end{abstract}

Key words: migration; family; health, women; Mexico

Este trabajo recibió el Segundo Premio en el Área de Investigación en Salud Pública y Ciencias Sociales otorgado por la Secretaría de Salud de México en el XI Encuentro Nacional de Investigadores, Acapulco, Guerrero, septiembre de 2006.

(I) Centro de Investigación en Sistemas de Salud, Instituto Nacional de Salud Pública. México.

(2) Facultad de Enfermería, Universidad de Antioquia. Colombia.

(3) Programa de Maestría en Antropología Social, Centro de Investigaciones en Estudios Superiores en Antropología Social, Occidente-Sureste. México.

(4) Programa de Doctorado en Antropología de la Medicina, Universidad Rovira iVirgili. Tarragona, España.

Fecha de recibido: 9 de noviembre de 2006 - Fecha de aceptado: 15 de noviembre de 2007 Solicitud de sobretiros: Dr. René Leyva-Flores. Instituto Nacional de Salud Pública.Av. Universidad 655, Santa María Ahuacatitlán, 62508 Cuernavaca, Morelos, México. Correo electrónico:rleyva@correo.insp.mx 
$\mathrm{E}_{\mathrm{s}}$ tudios sobre el acceso a servicios de salud han idenificado diversos factores estructurales que tienen especial repercusión en la búsqueda y utilización de servicios de salud por las mujeres. ${ }^{1}$ Entre estos factores se encuentran su condición económica, ${ }^{2,3}$ la relación con los proveedores de salud, ${ }^{4,5}$ la confianza en el sistema de atención médica ${ }^{6}$ y la representación del proceso saludenfermedad, ${ }^{7}$ entre otros. En estos estudios un elemento común es la inequidad de género como concepto explicativo del acceso diferencial a los servicios de salud, ${ }^{8}$ lo cual contrasta con el papel socialmente asignado a la mujer de cuidadora de la salud de los integrantes del grupo doméstico. ${ }^{9}$

Sin embargo, en México se ha documentado que las mujeres tienden a buscar atención médica antes y con mayor frecuencia que los hombres. ${ }^{10}$ No obstante, ante problemas de salud como las infecciones de transmisión sexual (ITS), que poseen un elevado componente de estigma social, el propio problema de salud se puede constituir en una barrera para la búsqueda oportuna de atención, ${ }^{11,12}$ que tiene especial relevancia en contextos sociales de alta migración, ${ }^{13}$ en los cuales se han identificado cambios en dos grandes componentes que relacionan el proceso de migración con la salud. ${ }^{14}$ Por una parte, se han documentado cambios en los comportamientos sexuales de los migrantes que constituyen factores de riesgo para la transmisión de ITS a sus parejas en los lugares de origen. Asimismo, en los ámbitos comunitario y familiar se han observado cambios en las relaciones, funciones y responsabilidades familiares y comunitarias, ${ }^{15,16}$ que pueden influir en la capacidad de las mujeres para buscar servicios de salud en las comunidades de origen.

La migración se ha estudiado desde diversas perspectivas de análisis de acuerdo con los diferentes enfoques disciplinarios de las ciencias sociales. La perspectiva de los hogares y la familia puede favorecer una mejor articulación entre los enfoques microsocial y macrosocial, ${ }^{17}$ sin perder de vista a su vez que la propia migración ha matizado conceptos clave en la definición de grupos domésticos. ${ }^{18}$ Puesto que la migración se ha constituido en un fenómeno estructural y se ha incrementado en los países en desarrollo, la salud de los migrantes se ha convertido en una preocupación no sólo para los lugares de destino, sino también para los de origen; ${ }^{19}$ en consecuencia, se espera que la relación entre migración y salud represente uno de los retos principales para la organización y funcionamiento de los servicios de salud, tema casi inexplorado en México. En este trabajo se analiza el vínculo entre la reestructuración del grupo familiar de las mujeres compañeras de migrantes en las comunidades de origen y la búsqueda de atención de servicios de salud, con el fin de aportar evidencias empíricas sobre la atención de la salud de este grupo de mujeres.

\section{Material y métodos}

Se realizó un estudio de carácter cualitativo de octubre de 2004 a julio de 2005 en dos comunidades, una urbana y otra rural, consideradas con alto índice de intensidad migratoria internacional. ${ }^{20}$ Como punto de partida se pensó que las comunidades, ubicadas en la región del centro de México, mostrarían diferencias en cuanto a composición familiar y búsqueda de atención de servicios de salud entre mujeres rurales y urbanas. Sin embargo, una vez obtenidos los datos, procesados y analizados, las diferencias no se establecían de acuerdo con el lugar de residencia de las mujeres y sus familias (rural o urbano), sino con el tipo de reestructuración del grupo familiar una vez que el compañero partía a Estados Unidos (EUA), es decir, si la mujer iba a vivir con su familia de origen o política o si se quedaba sola en el hogar que había formado con su compañero, factor que está relacionado con la edad de los hijos de la pareja.

En cada comunidad se seleccionó a mujeres que cumplieran con las siguientes características de inclusión: residentes de las comunidades, mayores de 18 años de edad, con pareja en EUA o cuya pareja hubiera regresado a la comunidad en un término no mayor de cinco años, y que aceptaran participar libremente en el estudio. Por lo tanto, la muestra quedó configurada por 60 mujeres compañeras de migrantes (28 de la comunidad rural y 32 de la comunidad urbana) que cumplieron con el principio de saturación teórica. ${ }^{21}$ Para entrar en contacto con las mujeres se recurrió a los servicios locales de salud, representaciones del gobierno local y a través de la técnica de bola de nieve en la cual una mujer compañera de migrante establecía el contacto con otra. A las participantes se les informó sobre el propósito del estudio y se obtuvo su consentimiento informado por escrito.

La recolección de los datos se realizó por medio de entrevistas en profundidad y de observación no participante. Las entrevistas en profundidad tuvieron como propósito explorar la reestructuración familiar y su relación con el proceso de búsqueda de atención a los servicios de salud sexual y reproductiva. La duración de las entrevistas fue de 60 a 70 minutos, en una o dos sesiones, las cuales fueron grabadas, transcritas y procesadas en el software Atlas-ti. ${ }^{*}$ Las entrevistas se efectuaron en el lugar en que las mujeres prefirieran. La observación no participante tuvo como objetivo contextualizar la interacción de estas mujeres con la comunidad; se registraron actividades en algunos lugares de encuentro donde se comparte la vida social (centro de

\footnotetext{
* Muhr T. Scientific Software Development. Visual Qualitative Data Version. ATLAS Ti 4.2. PC / Windows 95. Berlin: Scolari Sage Publications Software, 1997.
}

salud pública de méxico / vol. 50, no. 3, mayo-junio de 2008 
salud, pláticas del Programa Oportunidades, ${ }^{*}$ mercados y escuelas).

Para el análisis de los datos se tomó como base la aproximación interaccionista-simbólica. Dicha óptica retoma la dimensión del sentido subjetivo, rescata la acción social y asume que los actores se relacionan entre sí y con los objetos que les rodean en función del significado que tienen para ellos. ${ }^{22} \mathrm{El}$ procesamiento y análisis de los datos se llevó a cabo de acuerdo con las guías de la entrevista y su contenido, se incorporaron nuevos conceptos y categorías según lo requiriera el material y se clasificó la información por códigos. ${ }^{23} \mathrm{De}$ ese modo, las categorías de análisis fueron las siguientes: datos sociodemográficos; experiencia migratoria en la pareja y vivencias de la mujer; papel femenino, redes sociales y vivir la pareja; salud sexual y reproductiva; y utilización de servicios de salud.

Por último, cabe mencionar que antes de su inicio, este estudio pasó por la revisión y aprobación por parte de las comisiones de Investigación, Bioseguridad y Ética del Instituto Nacional de Salud Pública de México.

\section{Resultados}

\section{Perfil de las mujeres entrevistadas}

Más de la mitad de las mujeres compañeras de migrantes entrevistadas $(73 \%)$ nació fuera de las comunidades de estudio y provenía en su mayoría de comunidades rurales de estados cercanos; en general, esta migración tiene lugar en edades tempranas por decisión de sus progenitores, razón por la cual estas mujeres relacionan su pertenencia con el lugar de residencia actual. A pesar de ello, el fenómeno de la migración internacional ha estado presente a lo largo de su historia familiar, protagonizada por padres, hermanos, tíos o primos que se "iban al norte" a trabajar.

La edad de las mujeres es un factor que debe tenerse en cuenta a la luz de los resultados, ya que en términos generales se dividen en dos grupos: las que tienen entre 21 y 39 años de edad y las mayores de 40 años. Esto tiene relación no tanto con el número de hijos, sino con la edad de éstos; las mujeres del primer grupo de edad tienen

\footnotetext{
* Programa gubernamental creado para disminuir la pobreza y beneficiar a las familias en salud, educación y nutrición que ofrecen varias secretarías, entre ellas la de Salud y la de Educación. En este programa la norma compromete a las beneficiarias a asistir a pláticas de salud, en las cuales se les orienta sobre prevención de enfermedades y promoción de la salud y reciben un apoyo económico por cada hijo que estudia.
}

todavía a su cargo hijos menores, en comparación con las mujeres entrevistadas de más de 40 años, quienes tienen entre su descendencia hijos con edad suficiente para cuidar al resto de los hermanos. Esto se vincula a su vez con la reestructuración que se produce en la familia de dichas mujeres una vez que su compañero se desplaza a EUA. Las mujeres que se quedan solas son las que pertenecen a este segundo grupo: personas mayores de 40 años y con hijos mayores; y las mujeres que se integran a su familia de origen o a la política son las más jóvenes y con hijos todavía pequeños.

Más que destacar el estado civil de estas mujeres, que no representa diferencias en el tipo de compromisos entre la pareja y las familias por estar casada o vivir en unión libre, es importante reconocer la continuidad del vínculo de pareja que guardan estas personas con sus compañeros a pesar de la migración, es decir, con el hecho de que el varón pasa largos periodos lejos del núcleo familiar. Y esto es así porque casi todos estos compañeros son migrantes indocumentados: pagan a un intermediario por un viaje que se ha vuelto cada día más peligroso y cuyo éxito no siempre está asegurado. Esto hace alargar sus periodos de estancia en EUA, en donde no hay ciclos fijos de ida y retorno. Rosalinda, una mujer cuyo compañero ha ido a EUA en cuatro ocasiones explica:

\footnotetext{
Nosotros somos bien pobres y nacieron todos nuestros hijos en casitas de varitas como las de allá abajo [...] y esos fueron sus intentos de él para irse al norte; que sufrió mucho para irse al norte porque se fue, lo agarraron, lo metieron a la cárcel, lo volvió a intentar, lo volvieron a agarrar y ya se vino y ya pagó el dinero que debía aquí y se volvió a regresar, y se volvió a endrogar porque es de puro dinero que piden prestado como ora las primeras veces que se fue (MCM-CO23).
}

La pareja se mantiene si se logra conservar la comunicación, sea con visitas a la comunidad de origen del migrante, llamadas telefónicas, cartas y fotografías, entre otras, pero sobre todo con el envío de dinero, más o menos con regularidad. Todas las entrevistadas, excepto una, consideran que su pareja lo es porque mantiene comunicación y remite dinero. El único caso de una autodefinida "mujer separada" es el relato de un fenómeno ya descrito en la bibliografía, acerca de las "mujeres dejadas o abandonadas" a causa de la migración del varón, cuestión que supone otro tipo de problemática relacionada con la migración pero cuyo objeto no era el de esta investigación. ${ }^{24}$

Otro tema a destacar es el de la ocupación de estas mujeres y su relación con la migración del compañero. Poco más de la mitad de las mujeres entrevistadas se 
dedica de manera exclusiva al cuidado del hogar y los hijos, por lo que depende de las remesas que mandan sus compañeros de EUA. En las dos comunidades estudiadas se advierte una acentuada división del trabajo familiar, bajo un entendido social y cultural de que la mujer se dedica a la "esfera doméstica" y el compañero a la "esfera pública", con la tácita aceptación de que él es el exclusivo proveedor del hogar. En estas parejas en las que el varón migra se busca una mejoría en el interior de la familia, que no es alcanzable en la comunidad de origen. La migración ha cambiado el propio concepto de matrimonio o unión entre estas mujeres; si en un principio se basaba en la complementariedad de los trabajos del varón y la mujer, ahora el papel del compañero se reduce a ser suministrador de dinero enviado desde el norte. Sólo cuando a las mujeres no les alcanza con la cantidad remitida, entonces se dedican a vender comida preparada o ropa, o bien a emplearse en el servicio doméstico. Éstas son las tres ocupaciones más comunes entre las mujeres entrevistadas que realizan un trabajo extradoméstico ( $43 \%$ ). Hay que destacar en este punto de la manutención y economía familiares de estas mujeres que en su mayoría (73\%) reciben el apoyo del programa gubernamental Oportunidades. Es pertinente señalar que dicho programa se instituye en comunidades con graves índices de pobreza con la finalidad de beneficiar a las familias en salud, educación y nutrición. Las familias beneficiarias reciben una ayuda económica por cada hijo que estudie; además, este programa compromete a las madres de familia a asistir a pláticas de salud, en las cuales se las orienta acerca de la prevención de enfermedades y la promoción de la salud, en favor de una cultura saludable. Cabe destacar entonces que las dos comunidades, seleccionadas para esta investigación porque tienen un elevado índice de migración y se trata de comunidades expulsoras, son también comunidades en las que se opera un programa de este tipo para combatir la pobreza de su población (cuadro I).

\section{Reestructuración familiar}

La migración hacia EUA del compañero provoca una reestructuración en la composición de la familia de acuerdo con la presencia o ausencia del varón en la comunidad. En estas comunidades se pueden caracterizar dos tipos generales, si bien no únicos, que se reconocen en el momento en que el compañero parte hacia la Unión Americana: la integración de la mujer a su familia (sea la de origen o la política) y el caso de la mujer sola (cuadro II).

La primera se relaciona con el parentesco de la mujer o su compañero. El cambio de residencia ocurre en el momento en que su compañero deja la comunidad; entonces la mujer se traslada a casa de su familia de origen (padres o hermanos) o con la familia política (en casi todos los casos, los suegros). La decisión del cambio de residencia es del compañero, quien considera que estarán más "protegidos" en su ausencia. En este tipo se encuentra $51.6 \%$ de las mujeres y corresponde a las mujeres más jóvenes con hijos menores de 16 años (cuadro I), los cuales se convierten en el motivo principal para justificar el cambio. Se espera que la familia contribuirá a atenuar y satisfacer de mejor manera las necesidades de alimentación vestido y calzado de los hijos pequeños, además de ofrecer protección y compañía a la mujer que se queda sola en la comunidad. Las ayudas son económicas (préstamo de dinero mientras el compañero puede enviar dinero) o residenciales (alojamiento en el hogar de los familiares o préstamo de un cuarto en el mismo terreno). La asistencia también puede consistir en amparar a los hijos pequeños, comprar o cocinar y el aseo; la convivencia social y el apoyo moral y la protección (acompañamiento en determinadas ceremonias o rituales); y la información (consejos y orientaciones). La mayoría de estas mujeres lo percibe como un acto de intercambio y solidaridad entre los miembros de la nueva familia. Lilia, una de las mujeres más jóvenes entrevistadas que en fecha reciente se trasladó a vivir con sus suegros y cuñada, también compañera de migrante, afirma:

Apenas cuando él se fue y me dejó, me quedé con ellos, con sus papás porque pues yo tengo mi casa y yo vivía solamente con mi esposo, pero ahora que él se fue, pues me vine para acá con mis suegros para que no me sintiera sola $[. .$.$] y ahora que tuve la niña todos estuvieron en$ comunicación para saber cómo estaba y las dos partes de la familia me han apoyado mucho (MCM-CO18).

Se sienten protegidas y saben que tienen el respaldo de un amplio grupo de personas que le darán apoyo. Esto crea una red estable, además de ser accesibles por la cercanía física ya que todos viven en el mismo espacio y comunidad.

No obstante, esta protección supone también un mayor control de la familia en torno de decisiones, actividades, movimientos, distribución de las remesas enviadas por el compañero desde EUA, incluso en las formas de vestir y peinarse, así como en las interacciones de la propia mujer y sus hijos con la comunidad, ya que las decisiones no se toman de manera individual sino que prevalecen los intereses del grupo familiar. Azucena, una de las mujeres más jóvenes entrevistadas, sin hijos, señala lo siguiente:

A mi esposo no le gusta mucho ir a fiestas, pero a mí sí me gusta ir a los bailes;

Pregunta: Entonces ahora que no está él lo puedes hacer, ¿no?;

salud pública de méxico / vol. 50, no. 3, mayo-junio de 2008 


\section{Cuadro I}

Perfil sociodemográfico de las mujeres compañeras de migrantes entrevistadas DE ACUERDo CON LA RECOMPOSICIÓN FAMILIAR. MÉXICO, 2004-2005

\begin{tabular}{|c|c|c|c|c|}
\hline & & & & Total \\
\hline$=29$ & $48.33 \%$ & $n=31$ & $51.66 \%$ & $n=60$ \\
\hline
\end{tabular}

Edad

\begin{tabular}{|c|c|c|c|c|c|c|}
\hline 20 años o menos & - & - & 2 & $6.4 \%$ & 2 & 3.33 \\
\hline $21-29$ años & 4 & $13.8 \%$ & 10 & $32.2 \%$ & 14 & 23.33 \\
\hline 30-39 años & 8 & $27.6 \%$ & 13 & $41.9 \%$ & 21 & 35 \\
\hline 40-49 años & 12 & $41.3 \%$ & 5 & $16.1 \%$ & 17 & 28.33 \\
\hline 50 años o más & 5 & $17.2 \%$ & I & $3.2 \%$ & 6 & 10 \\
\hline
\end{tabular}

Lugar de nacimiento

\begin{tabular}{lllrrrrr} 
Lugar de residencia & 11 & $37.9 \%$ & 5 & $16.1 \%$ & 16 & 26.66 \\
\hline Fuera del lugar de residencia & 18 & $62.06 \%$ & 26 & $83.9 \%$ & 44 & 73.33
\end{tabular}

Escolaridad en años de estudio

\begin{tabular}{lrrrrrr} 
Menos de 6 años & 10 & $34.4 \%$ & 10 & $32.2 \%$ & 20 & 33.33 \\
\hline 7 años de estudio & 10 & $34.4 \%$ & 6 & $19.3 \%$ & 16 & 26.66 \\
\hline Entre 7 y 9 años de estudio & 5 & $17.2 \%$ & 11 & $35.4 \%$ & 16 & 26.66 \\
\hline 10 años y más & 4 & $13.7 \%$ & 4 & $12.9 \%$ & 8 & 13.33
\end{tabular}

Estado civil

\begin{tabular}{lcccccc} 
Casada & 21 & $72.4 \%$ & 25 & $80.6 \%$ & 46 & 76.66 \\
\hline Unión libre & 8 & $27.5 \%$ & 5 & $16.1 \%$ & 13 & 21.66 \\
\hline Separada / divorciada & - & - & 1 & $3.2 \%$ & 1 & 1.66
\end{tabular}

Número de hijos

\begin{tabular}{lrrrrrrr} 
Sin hijos & - & - & 1 & $3.2 \%$ & 1 & 1.66 \\
\hline I-2 & 9 & $31.03 \%$ & 8 & $25.8 \%$ & 17 & 28.33 \\
\hline $3-5$ & 16 & $55.1 \%$ & 14 & $80.6 \%$ & 30 & 50 \\
\hline 6 o más & 4 & $13.8 \%$ & 8 & $25.8 \%$ & 12 & 20
\end{tabular}

Ocupación

\begin{tabular}{lllllll} 
Sólo el hogar & 18 & $62.06 \%$ & 16 & $51.6 \%$ & 34 & 56.66 \\
\hline Hogar y trabajo extradoméstico & II & $37.9 \%$ & 15 & $48.3 \%$ & 26 & 43.33
\end{tabular}

Programa Oportunidades

\begin{tabular}{lrrrrrr} 
Sí & 21 & $72.41 \%$ & 23 & $74.19 \%$ & 44 & 73.33 \\
\hline No & 6 & $20.68 \%$ & 6 & $19.35 \%$ & 12 & 20 \\
\hline No hay datos & 2 & $6.89 \%$ & 2 & $6.45 \%$ & 4 & 6.66
\end{tabular}

Lugar de residencia

\begin{tabular}{lllllll} 
Rural & 16 & $55.1 \%$ & 12 & $38.7 \%$ & 28 & 46.66 \\
\hline Urbano & 13 & $44.8 \%$ & 19 & $61.2 \%$ & 32 & 53.33
\end{tabular}

Fuente: A partir de datos recogidos en el proyecto "Mujeres compañeras de migrantes y vulnerabilidad a ITS y VIH/SIDA", septiembre de 2004 a diciembre de 2006 


\section{Cuadro II \\ TIPOS RELACIONADOS CON LA RECOMPOSICIÓN FAMILIAR E IMPLICACIONES en los Procesos de búsQueda de Servicios de salud. Mujeres de migrantes. MéXico, 2004-2005}

\begin{tabular}{|c|c|c|}
\hline Tipos & Recomposición familiar & $\begin{array}{l}\text { Implicaciones para la búsqueda } \\
\text { de servicios de salud }\end{array}$ \\
\hline Integración a su familia o la del compañero & $\begin{array}{l}\text { - Mayor protección y control familiar } \\
\text { - Menor libertad } \\
\text { - Interiorización y reproducción del control familiar }\end{array}$ & $\begin{array}{l}\text { - Mayor capacidad de respuesta a problemas de } \\
\text { salud } \\
\text { - La familia se convierte en un mecanismo de } \\
\text { control que limita su libertad de decisión para } \\
\text { la búsqueda y acceso a servicios de salud sexual } \\
\text { y reproductiva }\end{array}$ \\
\hline Condición de mujer sola & $\begin{array}{l}\text { - Responsabilidad total de la familia (economía, hijos } \\
\text { cuidados y educación, otros familiares) } \\
\text { - Red social limitada, débil y no permanente: } \\
\text { - Mayor libertad } \\
\text { - Control social } \\
\text { - Estigmatización (disponible y dispuesta) }\end{array}$ & $\begin{array}{l}\text { - Menor capacidad de respuesta a problemas de } \\
\text { salud } \\
\text { - Mayor libertad de decisión para acudir a ser- } \\
\text { vicios de salud sexual y reproductiva }\end{array}$ \\
\hline
\end{tabular}

Fuente: Proyecto "Mujeres compañeras de migrantes y vulnerabilidad a ITS/VIH/SIDA" (2006)

Respuesta: Pues no mucho porque la suegra que me tocó es muy cabrona y nada más me anda cuidando donde quiera que voy $[\ldots]$.

P: ¿Y tú le pides permiso a tu esposo o tú te vas así o lo hablan?

R: Nada más le digo que tengo una fiesta, que si puedo ir, bueno o sea, no le pido exactamente permiso, pero sí le digo que sí voy a ir a una fiesta, pero siempre me dice que vaya con mi mamá que nunca vaya sola, pero sí voy sola (MCM-CO12).

En algunas de estas mujeres, sobre todo entre las más jóvenes, tiene lugar una interiorización y reproducción de ese control familiar, lo que representa una forma de ajuste a las reglas de comportamiento social en la comunidad: el deber ser y hacer de las mujeres; así aparecen expresiones como "Así se debe" o "Así está bien". Además, las mujeres también controlan sus propios movimientos cuando saben que las cuestionarán su compañero, la familia o la comunidad en relación con la vigilancia o la presión social. Fátima, una mujer de mediana edad madre de seis hijos, dice:

Los chismes de aquí llegan donde están ellos, si se hacen cosas supuestamente ocultas: si se hicieron, se sabe. Eso corre, tu marido se da cuenta [...] y por lo mismo que uno sabe de ellos de la gente que llega de allí y dice: "Pues fíjate que fulano por allá vive con otra vieja" o que la vieja de fulano tal o cual (MCM-CO16).
Por otro lado, en el segundo tipo caracterizado se encuentran $48.3 \%$ de las mujeres compañeras de migrantes entrevistadas, quienes permanecen con sus hijos en la vivienda que han conformado con su pareja al momento de la migración del compañero (cuadro I). Por lo general, estas mujeres están en otro periodo de su ciclo de vida (individual, de pareja y familiar), ya que son mujeres de mayor edad, con mayor experiencia migratoria de los ciclos migratorios del compañero, con hijos mayores de 16 años que las más de las veces son los que proporcionan apoyo a su madre.

Este hijito mío de 18 años salió del bachillerato y quería agarrar su carrera, pero su papá no pudo pues porque es mucho dinero el que me cobraban y ya no agarró su carrera mi niño.

Se quedó así, y él se quería ir con su papá, y me decía: "Mamá, ¿qué voy a estar haciendo aquí? Pues aquí no hay trabajo, no hay nada [...]" le digo: "No, m'ijo, tú eres el más grande aquí en la casa y yo me siento protegida por ti, le digo, tu papá no está y me siento contenta a dormir", porque él oye algo y se para [...] y por eso no dejo que se vaya (MCM-CO23).

En la mayoría de estos casos, la familia de origen, y algunas veces también la política, reside fuera de la localidad o no mantiene relaciones de solidaridad con la mujer. Cuando el compañero emigra, estas mujeres tienen que asumir la responsabilidad de la familia en 
aspectos como el económico (administra el dinero que el compañero remite, el que ella gana con su trabajo extradoméstico y, si es el caso, el que recibe del Programa de Oportunidades), además del cuidado y la educación de los hijos e incluso de otros familiares, como sus padres o suegros. Por lo regular, la red de apoyo de esta mujer se limita a un número pequeño de personas y se relaciona con la amistad, lazos afectivos y vecinos, pero la frecuencia de los intercambios es esporádica e inestable ya que no puede contar con esa ayuda o intercambios en toda ocasión. Fátima, de 49 años de edad, con seis hijos a su cargo, el mayor de 27 y el chico de 15 , comenta lo siguiente:

Aquí en esta colonia, como decía mi abuelita y con perdón tuyo, no hay quién te haga un favor porque aquí estamos todos jodidos; no hay quién tenga dinero, no hay quién al tener una grave necesidad, yo diga: "Pues voy con fulano a que me preste dinero", no hay eso aquí (MCM.CO16).

A pesar de esta situación se sienten con mayor libertad de movimientos y mayor poder de decisión porque nadie controla ni juzga sus entradas y salidas, tiempos, vestimenta y relaciones; estas personas sienten un control y estigmatización social como mujeres solas, ya que perciben que los hombres de la comunidad las ven como mujeres sexualmente disponibles, sin contar con que las demás mujeres de la comunidad las consideran fáciles o indignas, y aun una amenaza para sus matrimonios. Paulina, una de estas mujeres, reconoce que:

Aquí, tan pronto tu marido se va, se te acercan y te dicen [los hombres]: "Oye, si necesitas algo o lo que quieras y a la hora que quieras, estoy para servirte". O sea, que diciéndote otro tipo de cosas, ¿no? Y claro tú no eres tonta como para no entender (MCM-CO17).

\section{Implicaciones en los procesos de búsqueda de los servicios de salud}

La búsqueda de servicios de salud sexual y reproductiva en estas mujeres guarda una relación estrecha con estos tipos de reestructuración familiar y sus redes de apoyo (cuadro II).

Cuando la mujer se integra a su familia o la del compañero tiene mayor capacidad de respuesta a problemas de salud, tanto de los hijos, ella misma e incluso otros miembros de la familia. En este caso, la red es sólida en episodios de urgencias médicas y facilita y determina la decisión de buscar atención médica en momentos de incertidumbre. Los miembros de la familia también funcionan para consultar o pedir consejo.
Angélica, de 32 años y con dos hijos, y quien pasó a residir con sus suegros en ausencia de su compañero, indica lo siguiente:

P: ¿Qué tipo de problemas has tenido cuando tu marido anda por allá?

R: Bueno, pues en una ocasión a la niña le picó a las once de la noche un alacrán, y entonces empezamos a buscar taxi entre todos para llevarla al hospital de aquí, y con un piquete hay que correrle [...] y le dije a mi hermana: "Pues háblale a Ramón [su cuñado] porque a la niña le picó el bicho", y en seguida vino como a los cinco minutos y ya nos fuimos para el hospital (MCM-CO13).

La red se muestra amplia: la mujer sabe que ante una emergencia de salud cuenta con una serie de personas que la van a apoyar, ya sea que la acompañen, cuiden a sus otros hijos o se encarguen del transporte. Además, la red se vuelve estable y accesible ya que al vivir juntos o cerca es más fácil la solidaridad entre sus miembros.

En relación con la búsqueda de servicios de salud sexual y reproductiva de la mujer, la familia se convierte en un mecanismo de control. En el caso del uso de los métodos anticonceptivos se observa una presión familiar para que la mujer los suspenda cuando su compañero parte para EUA. Se entiende que su compañero debe ser su única pareja y que ella debe serle fiel, por lo que no se justifica el uso de métodos anticonceptivos. La búsqueda de atención también puede limitarse debido a que la suegra, la hermana, la madre o la cuñada la acompañan a la consulta, lo que dificulta la relación médico-paciente, sobre todo en la discusión de temas considerados "privados", por ejemplo la salud sexual y reproductiva de la mujer (anticoncepción o alguna infección de transmisión sexual).

Por otro lado, cuando la mujer se queda sola, tiene menor capacidad de respuesta a problemas de salud: en algún momento de crisis, la mujer no tiene a quién consultar sus dudas o inquietudes o pedir consejo. En consecuencia, la mujer toma la decisión de buscar atención. Las dificultades son diversas, desde no tener con quién dejar a los hijos hasta la imposibilidad de acudir al centro médico durante las entradas y salidas de los hijos a sus escuelas. Entonces recurre a las vecinas para que le echen la mano, ya que se trata de una eventualidad; por lo tanto, es frecuente que sus problemas de salud los resuelva mediante la automedicación o su desaparición espontánea, lo cual posterga la atención oportuna.

En el caso de la salud sexual, estas mujeres tienen mayor libertad de decisión para acudir a servicios de salud y continuar o no con los métodos anticonceptivos. Martina, de 40 años, con tres hijos, explica y argumenta sus decisiones: 
Con las pastillas (anticonceptivas) mi esposo se enojaba mucho [...] Con el niño me tardé, y se fue a Estados Unidos, y vino y me embaraza, y se vuelve a ir, y regresa y me embaraza [...] y con la última como no estaba él, el médico me preguntó si me operaba y le dije que sí [...] pero no, no se lo he dicho que estoy operada (MCM-C08).

La búsqueda de atención no está limitada, ya que al menos en el discurso estas mujeres expresan que pueden tomar sus propias decisiones y controlar el momento y la finalidad de buscar atención. Otras, las menos, toman decisiones cuando que el compañero se halla en EUA como forma de no crear tensión en la pareja.

\section{Puntos de coincidencia}

En ambas situaciones, ya sea que la mujer se integre a su familia o las mujeres solas, en la mayoría de las mujeres entrevistadas los embarazos se relacionan con la dinámica migratoria del compañero. Las mujeres quedan embarazadas en los meses en que su pareja está de regreso a la comunidad, pero siguen el embarazo y el parto sin él.

Aquí habemos familias que venían nada más, embarazaban a la mujer y daban la arrancada [se van a EUA] ya nada más venían a conocer a su hijo, que si a bautizarlo, o por equis razón se van quedando y al año o año y medio, de nuevo embarazada otra vez de vuelta la mujer, y se van y así pues hacen su vida (MCM-CO02).

El embarazo, casi necesario en cada regreso del compañero a la comunidad, y la oposición a la planificación por parte del compañero, son medidas para asegurar la fidelidad de la mujer en su ausencia. Además entre las mujeres compañeras entrevistadas no hay en la práctica diferencias en el tema de la anticoncepción, dado que suspenden o recuperan el uso de métodos anticonceptivos de acuerdo con las salidas y llegadas del compañero, por lo que se convierte en la muestra de esa fidelidad casi exigida. Ese sentir se acentúa con las ideas comunitarias de reciprocidad en estas parejas, en las que él se sacrifica al migrar y ella se queda al cuidado del hogar y los hijos sin caer en ninguna infidelidad.

P: ¿Y cuando su esposo no está, deja de usar la inyección (planificadora)?

R: Sí yo la dejo de usar [...] porque como él se tarda pues más de dos años en regresar, yo mientras descanso, ¿no? Y si regresa lo vuelvo a utilizar [...] porque él me dice cuándo viene, para yo esperarlo aquí (MCM-TEX13).
Además, la mayoría de las mujeres entrevistadas asume que el varón tendrá múltiples contactos sexuales en el camino y en el lugar de destino en EUA, mientras que ellas adoptan la abstinencia como parte de su papel de buena esposa. Paulina, una mujer de 30 años, con un hijo menor, dice al respecto:

P: ¿Y tú alguna vez has pensado que él haya tenido por allá relaciones [sexuales]?

R: Pues no sé, por qué no pensarlo. Como te digo, no estás ciega, no escapo de la realidad; si de pronto los hombres se van con algunos amigos a tomarse una cerveza y de pronto ven por ahí otra mujer, tú crees que son tontos como para decirte: "Sí, me fui a acostar con otra mujer"; o sea, no son tontos, nunca te lo van a decir, por muchas razones, pero es lógico que como hombres y machos, y en México lo son y bastante, lo van a hacer (MCM-CO17).

Por otro lado, todas las mujeres se encuentran con barreras de acceso a los servicios de salud de la comunidad, en particular en lo que corresponde a los tiempos y procedimientos burocráticos. El sistema no tiene en cuenta las dinámicas y horarios de las mujeres en relación con su familia. Asimismo, las mujeres expresan su insatisfacción porque los servicios de salud sólo están disponibles en la comunidad en horario matutino. En consecuencia, la medida a seguir, si disponen de dinero, es atenderse con un médico privado.

Además, una vez atendidas, deben comprar la mayor parte de las veces los medicamentos para el tratamiento, cuestión que afecta de forma negativa las economías familiares. En cuanto a su salud sexual y reproductiva, y puesto que la mayoría es beneficiaria del Programa Oportunidades, están comprometidas a someterse a una revisión ginecológica y Papanicolaou una vez al año; empero, en opinión de estas personas, tal práctica carece de utilidad porque los resultados son tardados (dos meses en promedio), por lo que es más un requisito formal que disipa además la idea de educación para la prevención.

Las mujeres cuyo Papanicolaou es dudoso deben desplazarse a la capital del estado por sus propios medios, a una hora y media de la comunidad rural y a unos 40 minutos de la comunidad urbana. En estas condiciones (tiempos, traslados, movimientos y adaptación a los nuevos lugares) se dificulta el acceso oportuno y la continuidad de las acciones médicas necesarias por el control adecuado de los casos. A ello se suma la burocratización del sistema que no da cuenta de los momentos de tensión por lo que pasa la mujer en estos procesos. Rosalinda, una mujer de la comunidad rural, de 41 años y con cuatro hijos, el más pequeño de cinco años, recuerda: 
R: Yo salí con síntomas de cáncer en la matriz y me mandan hasta la capital [...] me hizo unas cortadas y nomás el doctor no me dio explicación. Cuando yo llegué a mi casa, yo me sentía bien mal. Yo llegué llorando porque me dolía mucho. [...] y no me dijo nada, nada, y nada me dio $p a^{\prime} l$ dolor ni nada, y yo ya llevaba temperatura. Hasta que mi hermano me llevó al médico del centro de salud chiquito que tenemos aquí, y ése ya me explicó que no habían cortado nada, que eran puras quemaditas de las venitas y que tenía que cuidarme por unos días (MCM-CO23).

\section{Conclusiones}

El proceso migratorio en estas comunidades redefine las relaciones familiares y comunitarias que inciden sobre la capacidad de las mujeres para atender sus necesidades sociales, entre ellas las de salud. En este trabajo se han identificado dos tipos de reestructuración familiar, los cuales no tienen un carácter espontáneo ni responden a una libre decisión de la pareja, sino que dependen de la disponibilidad y el acceso a recursos familiares y comunitarios para responder a la salida del varón migrante. En estos cambios también modifican las relaciones de inequidad de género dentro de la familia, que marcan la vigilancia, el comportamiento de la mujer cuando su pareja sale de la comunidad y el control de la sexualidad de las compañeras de migrantes.

Si bien en las comunidades se pueden identificar estos dos grupos de mujeres, ambos no son excluyentes para un mismo grupo familiar ni para una misma mujer. De esta manera, en diferentes momentos la mujer puede incorporarse a la familia o manejar sola el proceso migratorio de su compañero; tener hijos pequeños es una condicionante para buscar apoyos familiares más cercanos y constantes.

En ambas comunidades (urbana y rural) las mujeres perciben diferentes formas de control social que limitan su capacidad de relacionarse con los integrantes de la comunidad, formas que se distinguen por la inequidad de género y los papeles tradicionales diferenciales entre mujeres y varones. Aun así, una parte considerable de estas personas ha asumido la responsabilidad de conducir el hogar y proveer los recursos materiales y económicos para el funcionamiento del hogar.

Estos cambios familiares tienen implicaciones contradictorias en la búsqueda de atención. Por una parte, para las mujeres que se integran a su familia o la del compañero hay mayor capacidad de respuesta ante problemas de salud por la movilización de los apoyos familiares, pero menor capacidad de decisión para seleccionar y demandar los servicios de salud que ella considera necesarios. Por otra parte, las mujeres que se quedan solas tienen menor capacidad de respuesta debido a la escasa red social de apoyo, pero cuentan con mayor autonomía para demandar servicios de salud.

La demanda de servicios de salud para ambos grupos de mujeres es compleja y con mayores efectos negativos para las mujeres solas que para las integradas a la familia. Desde la perspectiva de las mujeres de ambas comunidades, el uso de los servicios de salud requiere largos tiempos de espera; éstos se encuentran poco organizados en función de sus necesidades (están cerrados por las tardes, sábados y domingos), o bien es preciso desplazarse a otras comunidades que cuentan con unidades hospitalarias o servicios de mayor complejidad. Estos factores en su conjunto constituyen, desde la perspectiva de las mujeres, factores importantes que obstaculizan la demanda de servicios de salud.

\section{Discusión}

En estas comunidades, el proceso migratorio tiene un claro nexo con la potencial demanda de servicios de salud sexual y reproductiva. El uso de métodos anticonceptivos y el embarazo representan procesos que siguen los ciclos de migración, en los cuales la mujer tiene escaso control sobre el tipo y la temporalidad del uso de estos métodos. La decisión de suspender o continuar se toma casi siempre a petición del varón, pero también se asumen normas del comportamiento social esperado en estas mujeres. Por consiguiente, la ausencia del compañero supone que la mujer no necesita usar métodos anticonceptivos. Esto es una clara expresión del cumplimiento de la norma social de fidelidad de la mujer al varón, no así del hombre hacia la mujer. ${ }^{25}$

El uso de servicios de salud por procedimientos como el Papanicolaou, para la mayoría de las mujeres que participaron en este estudio, se encuentra como una condición para recibir los beneficios del Programa Oportunidades. El resultado de este procedimiento, considerado un suceso clave para la detección oportuna de problemas de cáncer cervicouterino y en algunos casos de diagnóstico de ITS, es tardío y se convierte, desde la perspectiva de las mujeres, en una obligación para continuar dicho programa más que en un elemento de beneficio a su salud. El caso documentado en esta investigación puede ser una muestra de los deficientes procesos de atención a la salud que implican gastos innecesarios y potencialmente catastróficos para la familia. ${ }^{26}$

Las mujeres entrevistadas enfrentan notables obstáculos para el acceso a los servicios de salud, desde elementos de reorganización familiar y comunitaria (control familiar para tomar decisiones en las mujeres que se integran a la familia y, para las que se quedan 
solas, escasa movilización de recursos familiares) o factores relacionados con la organización de los servicios (servicios no disponibles, escasa información que reciben ante sucesos concretos de salud sexual y reproductiva, entrega tardía de resultados de exámenes clave en la detección oportuna de problemas de salud) hasta los propios problemas de salud, como son las ITS que poseen un alto componente de estigmatización social. Estos obstáculos pueden ser comunes a otras mujeres que viven en diferentes comunidades de México; sin embargo, cobran especial relevancia en el contexto de alta migración, en la cual se refuerzan las inequidades de género y se reconfiguran las relaciones e interacciones familiares y comunitarias que funcionan como condicionantes de la búsqueda de servicios de salud.

El diseño de medidas para facilitar el acceso a los servicios de salud a las mujeres que viven en contextos de alta migración requiere tener en cuenta las condiciones y conductas familiares para manejar el proceso migratorio, reconocer la inequidad de género en las familias y la connotación social de problemas de salud como las ITS, con el fin de responder con mayor eficiencia y equidad a las necesidades de salud de estas mujeres.

\section{Agradecimientos}

Este trabajo es producto del proyecto de investigación denominado "Mujeres compañeras de migrantes y vulnerabilidad a ITS/VIH/SIDA" (Fondo Sectorial CONACyT-2003-057). Los autores agradecen la valiosa colaboración de las mujeres compañeras de migrantes que participaron en el estudio. También reconocen el apoyo técnico a Janette Flores Velásquez, estudiante de psicología del Centro Internacional de Estudios Superiores de Morelos, México, y el de Natasha Van Borek, estudiante de la maestría en salud pública de la Facultad de Ciencias de la Salud, Universidad Simon Fraser, Vancouver, Canadá.

\section{Referencias}

I.Nash-Durrenda G.Women access to health care in developing countries. Soc Sci Med 1992;35(4):613-617.

2.Barraza-Lloréns M, Bertozzi S, González-Pier E, Gutierrez JP.Adressing inequity in health care in Mexico. Health Affairs 2002; 21:47-56.

3. Sicchia S, Maclean H. Globalization, poverty and women's health: mapping the connections. Can J Public Health 2006; 97(I):69-7I. 4. Puentes-Markides C. Women and access to health care. Soc Sci Med 1992;35(4):619-626.

5. Bronfman M, Castro R, Zúñiga E, Miranda C, Oviedo J. Del "cuánto" al "por qué": la utilización de los servicios de salud desde la perspectiva de los usuarios. Salud Publica Mex 1997;39:442-450.
6. Humphreys J, Rolley F.A modified framework for rural general practice: the importance of recruitment and retention. Soc Sci Med 1998;46: 939-946.

7. Herek G, Capitanio J P,Widaman K F. Stigma, social risk and health policy: public attitudes toward HIVsurveillance policies and social construction of illness. Health Psychol 2003;22(5):533-540.

8. Gupta R. Gender, sexuality and HIVIAIDS: the what, the why, and the how. XIII International Conference Plenary Address, International Center for Research On Women 2000:I-8.

9. Castro R, Bronfman M. Teoría feminista y sociología médica: bases para una discusión. En Figueroa J. G, coord. La condición de la mujer en el espacio de la salud. México, DF: El Colegio de México, Centro de Estudios Demográficos y de Desarrollo Urbano, 1998:205-238.

10. Kaijzer B. El género y el proceso salud-enfermedad-atención. Gén Sal Cif 2003; I (3):3-7.

II. Herrera C, Campero L. La vulnerabilidad e invisibilidad de las mujeres ante elVIH/SIDA: constantes y cambios en el tema. Salud Publica Mex 2002;44(6):554-654.

12. Kendall T, Pérez. H. Hablan las mujeres mexicanas VIH positivas. Necesidades y apoyos en el ámbito médico, familiar y comunitario. México DF: Colectivo Sol,AC, 2004.

13. Magis-Rodriguez C, Gayet C, Negroni M, Leyva R, Bravo-García E, Uribe P, et al. Migration and AIDS in Mexico.An overview based on recent evidence.J Acquir Inmmune Defic Syndr 2004;37(4):S2I5-S226.

14. Bronfman M, Minello N. Hábitos sexuales de los migrantes a los Estados Unidos. Prácticas de riesgo para la infección porVIH. En: Bronfman M,Amuchastegui A, Martina R M, Minello N, Rivas M, Rodríguez G. SIDA en México. Migración, adolescencia y género. México: Colectivo Sol, 1995: I-89.

15. Bronfman M, Leyva-Flores R, Negroni F, Caballero M, Infante-Xibillé C, Cuadra SM, et al. Migración, género y SIDA: contextos de vulnerabilidad. Gen Sal Cif 2003; I (3):8-12.

16. Muñoz-Aguirre C. Impacto de la migración en la estructura y dinámica de los hogares. En: D. Barrera y C. Oehmichen (editoras). Migración y relaciones de género en México. México: Gimtrap,AC/UNAM, 2000: |57-|8|.

17. Massey D. Social structure, household strategies and the cumulative causation of migraction. Popul Index 1990;56(I):3-26.

18. D'Aubeterre-Buznego ME. Mujeres y espacio social transnacional: Maniobras para renegociar el vínculo conyugal. En: D Barrera, Oehmichen $C$, eds. Migración y relaciones de género en México. México: Gimtrap, AC/UNAM, 2000:65-85.

19. Consejo Nacional de Población. Migración México-Estados Unidos. Temas de Salud 2005: 34.

20. Consejo Nacional de Población. Índice de intensidad migratoria México-Estados Unidos; 2002: I8I.

21. Bertaux D. Los relatos de la vida en el análisis social. En:Aceves Lozano J, comp. Historia oral. México DF: Instituto de Investigaciones José María Mora, 1993:136-148.

22. Blumer H. Symbolic interactionism. Perspective and method. Englewood Cliffs, New Jersey: Prentice- Hall, 1969.

23. Strauss A, Corbin J. Bases de la investigación cualitativa técnicas y procedimientos para desarrollar la teoría fundamentada. Medellín, Colombia: Universidad de Antioquia, 2003.

24. Fagetti A. Mujeres abandonadas: desafios y vivencias. En: Barrera D, Oehmichen C, eds. Migración y relaciones de género en México. México: Gimtrap,AC/UNAM, 2000:119-134.

25. Hirsch J.A courtship marriage. Sexuality and love in mexican transnational families. Berkeley, EUA: University of California Press, 2003. 26. Sesma-Vázquez S, Pérez-Rico R, Sosa-Manzano CL, Gómez-Dantés O. Gastos catastróficos por motivos de salud en México: magnitud, distribución y determinantes. Salud Publica Mex 2005;47(supl I):S37-S46. 